APS/PRL

\title{
Mesoscale Organization and Dynamics in Binary Ionic Liquid Mixtures
}

\author{
Tyler Cosby and Joshua Sangord* \\ Department of Chemical and Biomolecular Engineering, \\ University of Tennessee, Knoxville, TN \\ Utkarsh Kapoor and Jindal K. Shah \\ School of Chemical Engineering, Oklahoma State University, Stillwater, OK
}

(Dated: May 9, 2019) 


\begin{abstract}
The impact of mesoscale organization on dynamics and ion transport in binary ionic liquid mixtures is investigated by broadband dielectric spectroscopy, dynamic-mechanical spectroscopy, x-ray scattering, and molecular dynamics simulations. The mixtures are found to form distinct liquids with macroscopic properties that significantly deviate from weighted contributions of the neat components. For instance, it is shown that the mesoscale morphologies in ionic liquids can be tuned by mixing to enhance the static dielectric permittivity of the resulting liquid by as high as $100 \%$ relative to the neat ionic liquid components. This enhancement is attributed to the intricate role of interfacial dynamics associated with the changes in the mesoscopic aggregate morphologies in these systems. These results demonstrate the potential to design the physicochemical properties of ionic liquids through control of solvophobic aggregation.
\end{abstract}

Elucidating the influence of mesoscale organization on the dynamics and transport properties of ionic liquids is critical to developing design criteria for their applications in chemical synthesis, nanoparticle growth, biomass processing, batteries, solar cells, and supercapacitors. [1-10] In the past decade, the formation of mesoscale polar and non-polar domains in ionic liquids with substantial non-polar, alkyl side groups was recognized in detailed x-ray scattering, neutron scattering, and molecular dynamics (MD) simulation studies. [11 16] Mesoscale organization has been used to qualitatively explain numerous experimental findings which imply spatially and temporally distinct regions within bulk ionic liquids.[11, 17 26] Recent studies suggest that the existence and dynamics of the aggregates in neat ionic liquids, associated with fluctuations of the polar and non-polar regions, correlate strongly with many of the physicochemical properties of ionic liquids including transport properties such as zero-shear viscosity, dc ionic conductivity, and static dielectric permittivity. 27 32] However, no efforts to exploit the mesoscale organization to design novel ionic liquids with unique physical and chemical properties have been reported. In this work, it is demonstrated that by mixing ionic liquids with varying degrees of solvophobic aggregation, it is feasible to design distinct liquids with macroscopic properties that significantly deviate from weighted contributions of the neat components.

The local organization, or morphology, of the mesoscale aggregates is in part determined by the relative volume fractions of the polar and non-polar groups of the component ions.[11] In amphiphilic imidazolium, pyrrolidinium, piperidinium, quaternary phos- 
phonium, and quaternary ammonium ionic liquids, increasing the alkyl chain length on the cation headgroup tends to swell the non-polar domain leading to a progression from globular morphology to a loosely-defined bicontinuous morphology with percolating polar and non-polar domains. [13, 14, 33, 34] Conversely, at a given length of the alkyl chain on the cation, the polar domain may be enlarged (or reduced) by selecting an anion with a larger (or smaller) molar volume.[11] An alternative approach to altering the polar and non-polar volume fractions is to consider mixtures of two or more ionic liquids with differing chemical structures. 29] We hypothesize, for instance, that in an amphiphilic ionic liquid mixed with a predominantly polar ionic liquid, which preferentially locates within the polar domain, the polar versus non-polar volume fraction and accordingly the morphology can be tuned by simply altering the composition. Any number of ion chemical structure combinations can be envisioned for the mixture components. Additional and more complex morphologies can be accessed by mixing ionic liquids with differing chain lengths as well as other chemical structure features. [29] Totally unexplored is the influence of composition-dependent morphology and the accompanying mesoscale aggregate dynamics on the physical and chemical properties of ionic liquid mixtures. Unraveling this interplay requires the systematic investigation of well-chosen mixtures by a combination of techniques capable of probing mesoscale aggregate morphology, ion and mesoscale aggregate dynamics, as well as the bulk or macroscopic transport properties.

In this study, we highlight an approach in which complementary experimental and computational techniques are employed to investigate changes to mesoscale aggregate morphology and dynamics as a function of composition in binary mixtures of the ionic liquids 1-octyl3-methylimidazolium tetrafluoroborate $\left(\mathrm{C}_{8} \mathrm{MIm} \mathrm{BF}_{4}\right)$ and 1-ethyl-3-methylimidazolium tetrafluoroborate $\left(\mathrm{C}_{2} \mathrm{MIm} \mathrm{BF}_{4}\right)$. We find that by mixing these two imidazolium ILs, which differ only in the cationic alkyl chain length, we can transform the bicontinous morphology of neat $\mathrm{C}_{8} \mathrm{MIm} \mathrm{BF}_{4}$ to more isolated and spherical non-polar aggregates as indicated by x-ray scattering and molecular dynamics simulations. As a result of the composition-dependent evolution in morphology, the mesoscale aggregate dynamics, as probed by dynamic-mechanical and broadband dielectric spectroscopy, are significantly altered. The changes to aggregate morphology and dynamics result in a $100 \%$ increase in the static dielectric permittivity, also known as the dielectric constant, relative to that of either pure component. 
The chemical structures of $\mathrm{C}_{8} \mathrm{MIm} \mathrm{BF}_{4}$ and $\mathrm{C}_{2} \mathrm{MIm} \mathrm{BF}_{4}$ were chosen to approximate the desired mixture consisting of one amphiphilic and one predominantly polar ionic liquid. The imidazolium head group and the anion of each ionic liquid is identical in order to minimize the potential influence of mixture composition on the ion-ion interactions within the polar domains and at polar/non-polar interfaces. In this way, the influence of mesoscale aggregate morphology and dynamics on the transport properties may be investigated independent of any change in ion-ion interactions. To probe the influence of composition on the mesoscale aggregate morphology, and to verify the location of $\mathrm{C}_{2} \mathrm{MIm} \mathrm{BF}_{4}$ within the polar domain, neat $\mathrm{C}_{8} \mathrm{MIm} \mathrm{BF}_{4}$, neat $\mathrm{C}_{2} \mathrm{MIm} \mathrm{BF}_{4}$, and 30,50 , and $70 \mathrm{~mol} \% \mathrm{C}_{2} \mathrm{MIm} \mathrm{BF}_{4}$ mixtures were investigated by x-ray scattering and molecular dynamics simulations.

The structure factors, $\mathrm{S}(\mathrm{q})$, of the neat ionic liquids $\mathrm{C}_{8} \mathrm{MIm} \mathrm{BF}_{4}$ and $\mathrm{C}_{2} \mathrm{MIm} \mathrm{BF}_{4}$ and the 30,50 , and $70 \mathrm{~mol} \% \mathrm{C}_{2} \mathrm{MIm} \mathrm{BF}_{4}$ mixtures obtained at room temperature by small- and wide-angle x-ray scattering are presented in Figure 1(a). $\mathrm{C}_{8} \mathrm{MIm} \mathrm{BF}_{4}$ exhibits a pre-peak at $\mathrm{q}=0.28 \AA^{-1}$ typical of self-assembled ionic liquids and assigned to the scattering from polar domains separated by a non-polar domain. [11] The higher q-peak arises from adjacency correlations of both inter- and intramolecular origins and is common to all ionic liquids. [17] $\mathrm{C}_{2} \mathrm{MIm} \mathrm{BF}_{4}$ has no pre-peak and is therefore taken to be non-aggregating. With increasing concentration of $\mathrm{C}_{2} \mathrm{MIm} \mathrm{BF}_{4}$ the pre-peak is reduced in intensity and shifts to slightly lower q-values. Insight into the structural changes which alter the position and intensity of the pre-peak is provided by complementary MD simulations. Details of the structure factors calculated from the MD simulations may be found in the Supplementary Information. The structure factors, shown in Figure 1(b), reproduce the positions and relative intensities of the experimental structure factors reasonably-well over the entire q-range. The real space distances corresponding to the pre-peak, $d=2 \pi / q_{\max }$, found by experiments and simulations are presented in the inset of Figure 1(b). MD simulations slightly over-predict the experimental values, however, the non-monotonic dependence of the domain distance on composition is well-reproduced. The excellent agreement between MD simulation and experimental results provides confidence in the assignment of certain composition-dependent morphological transitions which are described by the subsequent detailed analysis of the MD simulations.

The connectivity of the nano-segregated polar/non-polar structure is examined in a quantitative manner in terms of domain analysis based on Voronoi tessellation technique. [35] In 


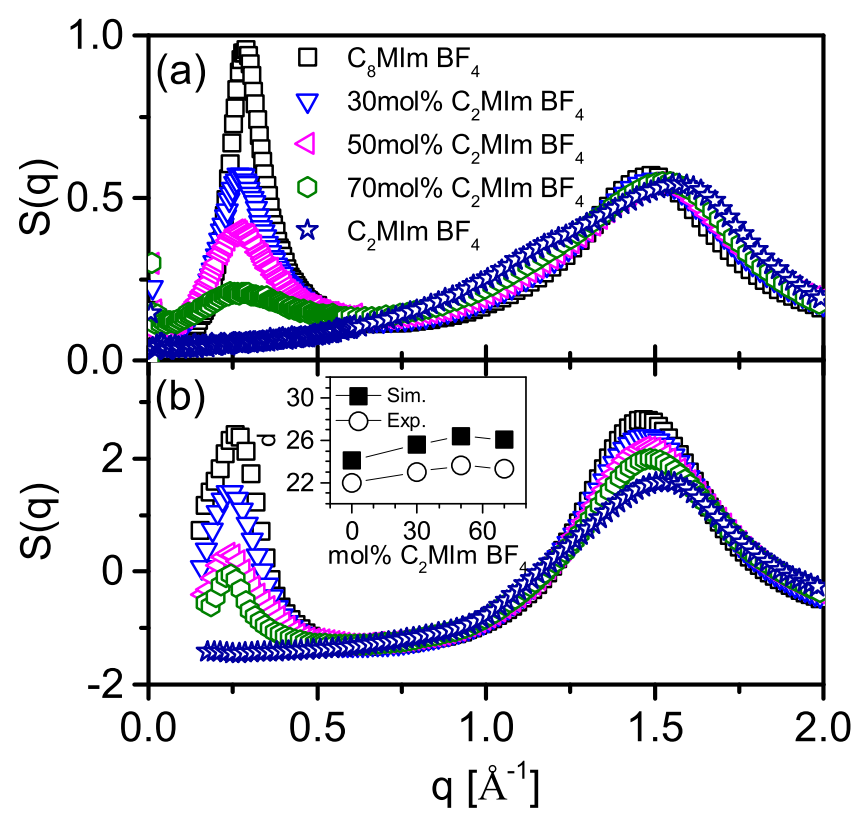

FIG. 1. (a) Structure factors, S(q), obtained by x-ray scattering. (b) Structure factors computed by MD trajectories. Inset: Comparison of the real-space correlation distance, $d=2 \pi / q_{\max }$, of the pre-peak obtained by simulation (sim.) and experiment (exp.).

this analysis, adjacent Voronoi cells sharing a face and belonging to a given subunit constitute a domain. For our purposes, each of the binary ionic liquid mixture systems is characterized in terms of four unique domains: (a) the total polar domain composed of the polar groups of both the cations and the anion; (b) $\mathrm{C}_{8} \mathrm{MIm}$ non-polar; (c) $\mathrm{C}_{2} \mathrm{MIm}$ non-polar and (d) total non-polar containing the non-polar groups from both cations. The polar group of both imidazolium cations contains the imidazolium ring as well as the methyl and methylene groups directly bonded to the ring, while the anion is completely polar. The polar group of the cation and anion together constitutes the overall polar domain. The non-polar regions in the two cations are the respective uncharged carbon groups minus the methylene group directly bonded to the imidazolium ring. The uncharged alkyl chain of the cations are considered unique in order to identify the origin of the structural changes at various concentrations. Figure 2(a) provides number of domains based on this classification as a function of the $\mathrm{C}_{2} \mathrm{MIm} \mathrm{BF}_{4}$ concentration. As expected, a domain count of 1 is observed for the polar domain indicating its three-dimensional connectivity for all the ionic liquids mixtures studied here. This observation is in line with previous simulation studies involving 


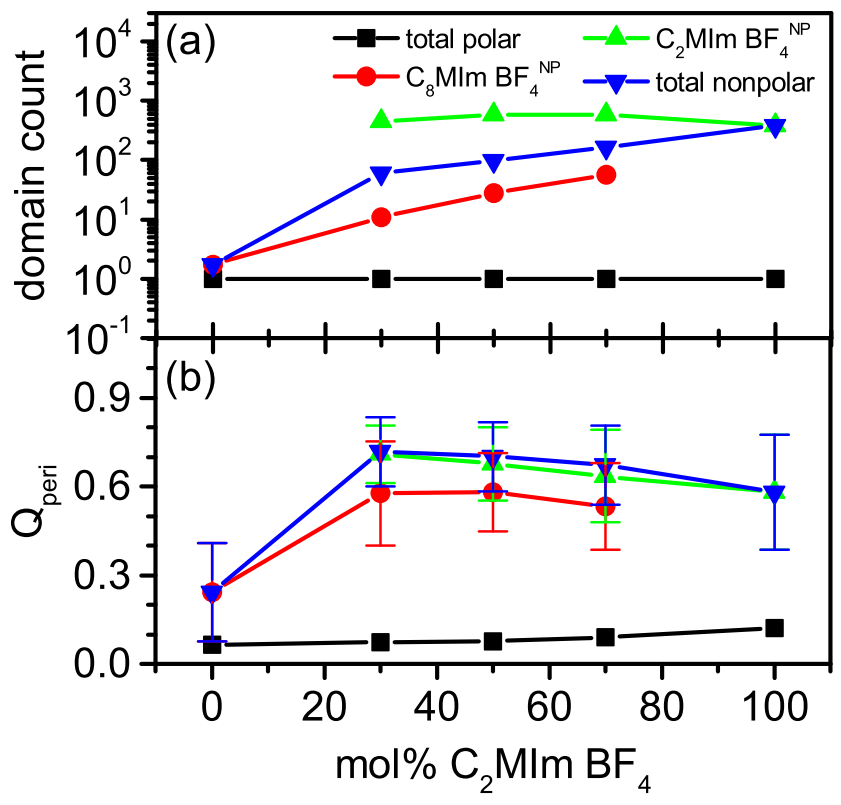

FIG. 2. (a) Average domain count of the polar and non-polar domains present in the simulation box as a function of $\mathrm{C}_{2} \mathrm{MIm} \mathrm{BF}_{4}$ concentration. (b) Average isoperimetric quotient, $\mathrm{Q}_{\mathrm{peri}}$, of respective cation/anion polar and nonpolar domains as a function of $\mathrm{C}_{2} \mathrm{MIm} \mathrm{BF}_{4}$ concentration. Please note that average numerical value and standard deviations were computed by dividing the trajectory into three blocks.

a wide range of pure ionic liquids. [11, 15, 16, 33, 35 39] For pure $\mathrm{C}_{2} \mathrm{MIm} \mathrm{BF}_{4}$, the domain counts for the non-polar group are significantly higher than $1(\sim 380)$ indicating that the cation non-polar groups are dispersed in the system. On the other hand, the domain count for the non-polar tails in the pure $\mathrm{C}_{8} \mathrm{MIm} \mathrm{BF}_{4}$ ionic liquid is between 1 and 2, indicating that the majority of alkyl chains are connected in a single percolated non-polar domain with some possible occurrence of isolated $\mathrm{C}_{8} \mathrm{MIm} \mathrm{BF}_{4}$ non-polar chains. The addition of $30 \mathrm{~mol} \%$ $\mathrm{C}_{2} \mathrm{MIm} \mathrm{BF}_{4}$ results in a significant disruption of the non-polar connectivity as the large single continuous domain is broken into as many as 10 separate domains and the number reaches as high as 57 at the highest $\mathrm{C}_{2} \mathrm{MIm} \mathrm{BF}_{4}$ concentration.

A quantitative metric of the variety of shapes adopted by the polar and non-polar domains is provided by the isoperimetric quotient, $Q_{\text {peri }}=\left[r_{\text {sphere }}(V) / r_{\text {sphere }}(A)\right]^{6}=36 \pi\left[V^{2} / A^{3}\right]$, where $V$ and $A$ denote the volume and area of a given domain respectively while $r_{\text {sphere }}(V)$ and $r_{\text {sphere }}(A)$ represent the equivalent radii of the sphere with volume $V$ and the sphere 
with area $A$, respectively. With this definition, the shape parameter will assume a value of 1 for a perfectly spherical shape while any deviations from sphericity lead to the values lower than 1. 39] The change in the isoperimetric quotient as a function of the $\mathrm{C}_{2} \mathrm{MIm} \mathrm{BF}_{4}$ concentration is shown in Figure 2(b). From the figure, it is clear that $Q_{\text {peri }}$ for the polar domain shows a negligible dependence on the concentration of $\mathrm{C}_{2} \mathrm{MIm} \mathrm{BF}_{4}$ and is always less than 0.1 , which implies that the shape of the polar network differs greatly from sphericity. Further, the non-polar domain present in pure $\mathrm{C}_{8} \mathrm{MIm} \mathrm{BF}_{4}$ ionic liquid has a $\mathrm{Q}_{\text {peri }}$ value less than 0.25 , and domain count of approximately 1, suggesting a network whose shape is also far from spherical. However, with the introduction of $30 \mathrm{~mol} \%$ of $\mathrm{C}_{2} \mathrm{MIm} \mathrm{BF}_{4}$ in $\mathrm{C}_{8} \mathrm{MIm}$ $\mathrm{BF}_{4}$, the $\mathrm{Q}_{\text {peri }}$ value nearly doubles assuming a value of $\sim 0.58$ suggesting a transition in the morphology of the domains which now more closely resemble a sphere in comparison to that in the pure $\mathrm{C}_{8} \mathrm{MIm}_{\mathrm{BF}}$. The results are even more dramatic when the total non-polar domain of the mixture is considered with values approaching as high as 0.7 at $30 \mathrm{~mol} \%$ $\mathrm{C}_{2} \mathrm{MIm} \mathrm{BF}_{4}$. Above $30 \mathrm{~mol} \%$, the $\mathrm{Q}_{\text {peri }}$ is practically composition independent, indicating that the transition in mesoscale aggregate shape occurs at or below this concentration. We conjecture that the dispersed subphase of $\mathrm{C}_{2} \mathrm{MIm}$ modulates the overall non-polar domain connectivity and morphology in a way that alters the dynamics of the mesoscale aggregates and gives rise to a variation in the static dielectric permittivity as a function of $\mathrm{C}_{2} \mathrm{MIm}_{\mathrm{BF}}$ concentration, as discussed later in the current work.

To probe the influence of composition-dependent morphology on the mesoscale aggregate dynamics, the neat ionic liquids and their mixtures were investigated by dynamic-mechanical and broadband dielectric spectroscopy. These data are presented in Figure 3 in terms of the imaginary part of complex viscosity, $\eta^{\prime \prime}$, and the derivative representation of the dielectric loss, $\varepsilon_{d e r}^{\prime \prime}$, respectively. Two distinct relaxation peaks, well separated in frequency, are observed in each experiment. The composition-dependent calorimetric glass transition temperatures, $\mathrm{T}_{g}$ 's, obtained by differential scanning calorimetry, follow a weighted average of the two neat IL components. The rates of the faster, higher-frequency mechanical and dielectric relaxations scale by $\mathrm{T}_{g}$. This comparison indicates that, in both experiments, the faster relaxations arise from the same underlying ion dynamics which define the glass transition in this class of ionic liquids.[30, 40] By comparison, the slower dielectric relaxation rate, $\omega_{\text {slow, } B D S}$, has a more complex composition dependence, as discussed later, indicating it is not directly associated with the glass transition as was recently suggested. [41] Further 


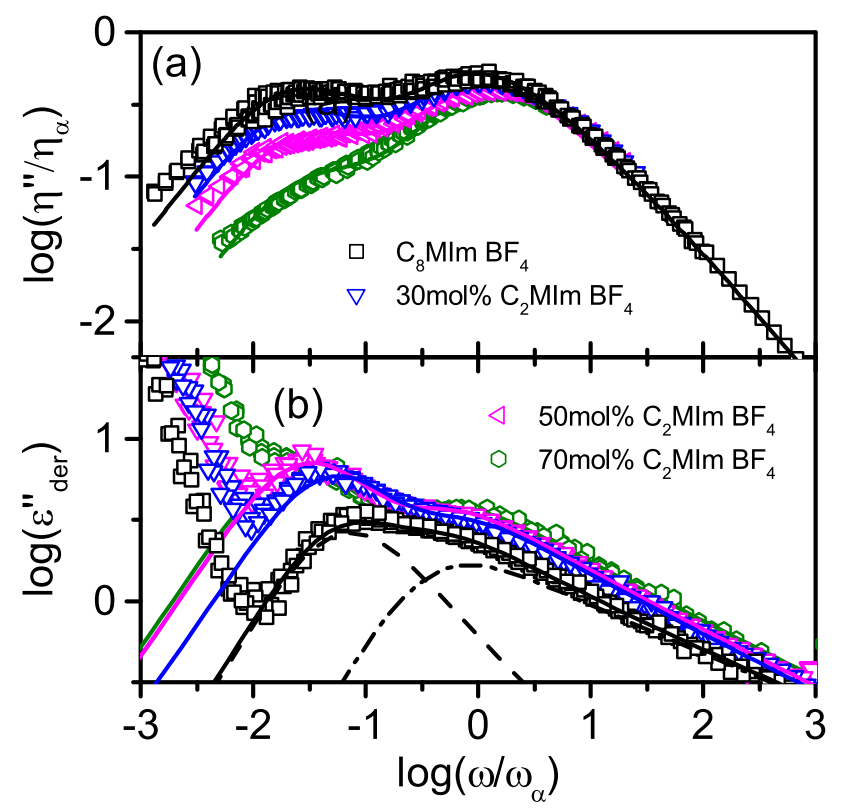

FIG. 3. (a) Imaginary part of complex viscosity, $\eta^{*}=\eta^{\prime}-i \eta^{\prime \prime}$, normalized by the structural relaxation contribution to the zero-shear viscosity, $\eta_{\alpha}=G_{\infty} / \omega_{\alpha}$, versus frequency normalized by the structural relaxation rate, $\omega_{\alpha}$. Solid lines correspond to the total fit by two Cole-Davidsonmodified Maxwell relaxation models. (b) The derivative representation of the dielectric loss, $\varepsilon_{d e r}^{\prime \prime}$. $\mathrm{T}=204-228 \mathrm{~K}$ at $8 \mathrm{~K}$ increments. Solid lines correspond to the total fit of two Havriliak-Negami fit functions at $204 \mathrm{~K}$. The dashed and dotted-dashed lines represent the separate Havriliak-Negami functions of the underlying slow and structural relaxations, respectively, for $\mathrm{C}_{8} \mathrm{MIm} \mathrm{BF}_{4}$. Details of the fitting functions and their parameters are provided in the Supplementary Information.

details on the $\mathrm{T}_{g}$ 's and temperature-dependent relaxation rates are presented in the Supplementary Information. In order to emphasize the influence of composition on the slower mesoscale dynamics, the spectra in Figure 3 are shown versus frequency normalized by the rate of the structural relaxation, $\omega_{\alpha}$. In this representation, it is evident that an additional relaxation is present in each experiment at rates slower than that of the primary structural relaxation in agreement with prior studies. [27, 42, 43]

In neat $\mathrm{C}_{n} \mathrm{MIm} \mathrm{BF}_{4}$ ionic liquids, the emergence of the slow dynamics was found to coincide with the onset of solvophobic aggregation, as evidenced by the development of the x-ray scattering pre-peak, and by a comparison between the relaxation rates with those previously obtained by neutron spin echo spectroscopy. 27, 29 31] Therefore, the slow relax- 
ations were attributed to fluctuations of the mesoscale aggregates at timescales longer than the structural relaxation. This attribution is further substantiated by Yamaguchi's recent computational work which shows that a cross-correlation exists between the shear stress relaxation and the slow relaxation of the domain structure corresponding to the scattering pre-peak. 28] It should also be noted that similar slow, sub-structural relaxations are also observed in some other mesoscopically ordered liquids, most notably monohydroxy alcohols, where they are also attributed to a supramolecular origin. 44 49] Despite the apparent similarities in the dielectric and dynamic-mechanical spectra, these techniques are sensitive to distinctly different correlations within the bulk liquid, i.e. polarization and the stress tensor, respectively. Therefore, a careful comparison of the influence of composition on the strength and rate of the slower mesoscale aggregate dynamics, as obtained by each technique, may provide a useful insight into its molecular origin.

Upon dilution of $\mathrm{C}_{8} \mathrm{MIm} \mathrm{BF}_{4}$ with $\mathrm{C}_{2} \mathrm{MIm} \mathrm{BF}_{4}$, the slow mechanical mesoscale relaxation is gradually reduced in strength until at $70 \mathrm{~mol} \% \mathrm{C}_{2} \mathrm{MIm} \mathrm{BF}_{4}$ it is barely visible as a low-frequency shoulder to the structural relaxation. However, relative to the structural relaxation rate, the rate of the slow relaxation is independent of composition. This trend is in stark contrast to observations of the slow dielectric relaxation. Upon addition of $\mathrm{C}_{2} \mathrm{MIm}$ $\mathrm{BF}_{4}$, the slow dielectric relaxation substantially increases in strength and shifts to lower frequencies relative to the structural, $\alpha$-relaxation rate. The relaxation rates and strengths are provided in the Supplementary Information. The divergence in the composition dependence of the slow relaxations probed by each technique indicates a possible sensitivity of the dielectric relaxation mechanism to the mesoscale aggregate shape or morphology which is lacking in the mechanical relaxation.

Several factors could potentially influence the mesoscale aggregate dynamics such as the composition-dependent volume fraction, shape, and size of the aggregated non-polar domains as well as any alteration in ion-ion interactions at the polar/non-polar interfaces. By our choice of cation and anion for the two ionic liquid components we have attempted to minimize the latter effect and will not consider it further. The size of the non-polar domains is probed by the real-space correlation distance corresponding to the x-ray scattering pre-peak. These distances, given in the inset of Figure 1 (b), increase with increasing concentration of $\mathrm{C}_{2} \mathrm{MIm} \mathrm{BF}_{4}$. However, the modest increase in aggregate dimensions by $\approx 2 \AA$ is not considered sufficient to explain the substantial changes in aggregate dynamics observed in 


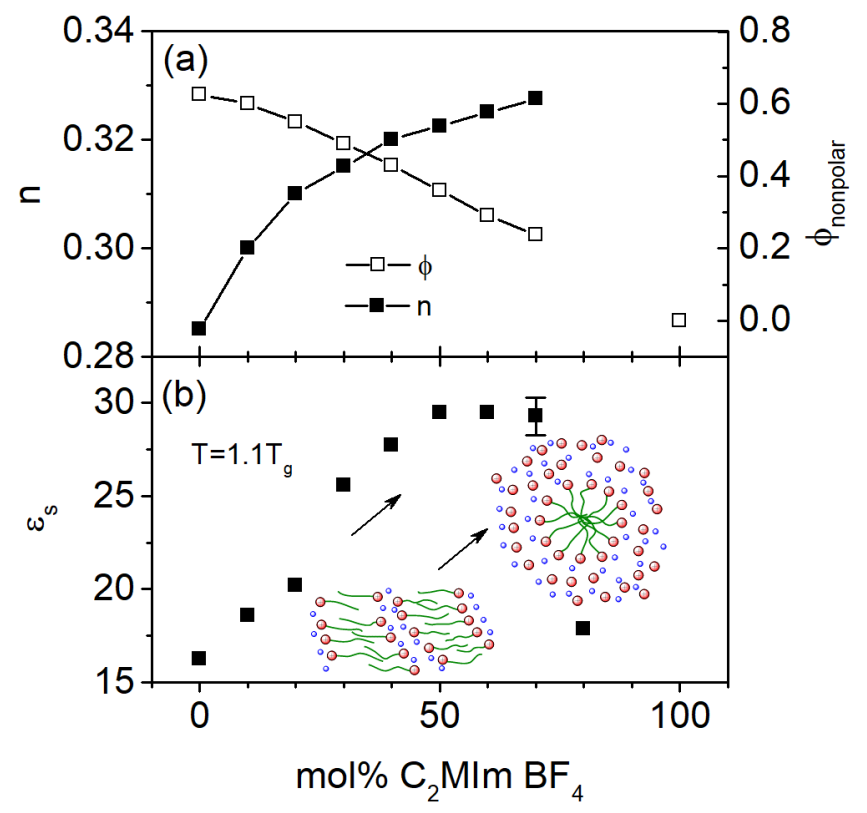

FIG. 4. (a) Shape parameter, n, and volume fraction of the non-polar phase, $\phi_{\text {nonpolar }}$, versus mol\% $\mathrm{C}_{2} \mathrm{MIm} \mathrm{BF}_{4}$. (b) Concentration dependence of static dielectric permittivity at $\mathrm{T}=1.1 \mathrm{~T}_{g}$. The increase in $\varepsilon_{s}$ is due to the concentration-dependent aggregate shapes illustrated by the inset cartoons.

the mechanical and dielectric spectra. We also note the relative invariance of the mechanical mesoscale aggregate relaxation rate as evidence for this relatively minor change in nonpolar domain size. The gradual reduction in the strength of the slow mechanical relaxation is consistent with a reduction in volume fraction of the non-polar aggregate domains in which it originates. Accordingly, the opposite composition-dependence of the slow dielectric relaxation strength suggests an overriding influence of aggregate shape rather than volume fraction.

It is proposed that the slow dielectric relaxation originates from interfacial polarization at the polar/non-polar interfaces. From numerous studies on heterogeneous liquids and solids it is well established that interfacial polarizations are strongly dependent on the shapes of the included domains. 50 53] A change in the shapes of the aggregates might therefore be the origin of the observed increases in strength of the slow relaxation. The influence of a transition in aggregate shape on interfacial polarization can be ascertained using an effective medium approximation (EMA). EMAs are useful approximate approaches to relate 
the shape and volume fractions of a filler phase located within host matrices to the macroscopic dielectric properties of the composite, provided the properties of the two phases can be estimated.[53 [56] Insight into the interplay of aggregate shapes, volume fractions, and dielectric relaxation strengths may be obtained by probing the ability of an EMA to accurately predict the static dielectric permittivities, $\varepsilon_{s}$, and dc ionic conductivities, $\sigma_{0}$, of the mixtures. These values are defined as the low-frequency limiting values of the real parts of complex dielectric permittivity and complex conductivity, respectively, see Supplementary Information. [55] The static dielectric permittivity, $\varepsilon_{s}=\Delta \varepsilon_{\text {slow }}+\Delta \varepsilon_{\alpha}+\varepsilon_{\infty}$, contains contributions from all higher frequency dielectric relaxations including the structural and slow dielectric relaxation strengths, $\Delta \varepsilon_{\alpha}$ and $\Delta \varepsilon_{\text {slow }}$, as well as other processes included in the high-frequency limiting permittivity, $\varepsilon_{\infty}$. In the ionic liquid mixtures, the composition dependence of $\varepsilon_{s}$ is dominated by and is roughly proportional to the strength of the slow dielectric relaxation. For our investigation, we employ a form of the symmetric Looyenga equation [54], which is suitable for the conducting phases and intermediate volume fractions found in our IL mixtures. [54] Details of this EMA and our application of it may be found in the Supplementary Information. The two fit parameters of this model, $n$ and $\phi$, correspond to the shape factor and volume fraction of the non-polar domain, see Figure 4(a). The experimental values of $\varepsilon_{s}$ and $\sigma_{0}$ are predicted only by an increase in $n$ and a concomitant decrease in $\phi$. Due to the assumptions on which an evaluation of the EMA relies, these trends can only be interpreted qualitatively. The overall reduction in volume fraction of the non-polar domain is consistent with its dilution upon addition of the non-aggregating $\mathrm{C}_{2} \mathrm{MIm} \mathrm{BF}_{4}$. The shape factor, $n$, is related to the shape of the insulating phase, with $n$ $<1 / 3$ corresponding to rod-like inclusions and $n=1 / 3$ to spherical.[53, 54] The gradual increase in $n$, and accordingly $\varepsilon_{s}$, is consistent with a transition to more spherical mesoscale aggregates. The majority of the increase in $\varepsilon_{s}$ occurs over the $20-30 \mathrm{~mol} \% \mathrm{C}_{2} \mathrm{MIm} \mathrm{BF}_{4}$, with a plateau above $\approx 40 \mathrm{~mol} \% \mathrm{C}_{2} \mathrm{MIm} \mathrm{BF}_{4}$ implying that the non-polar domain continues to retain sphere-like morphology above this minimum concentration.

Due to the close agreement between the trends found by MD simulation, dynamic mechanical spectroscopy, and dielectric spectroscopy, we attribute the increase in $\varepsilon_{s}$, Figure 4 (b), and accordingly the strength of the slow dielectric relaxation to a transition in the mesoscale aggregate morphology owing to the dilution of non-polar domains upon addition of $\mathrm{C}_{2} \mathrm{MIm}_{\mathrm{BF}_{4}}$. As a direct result of the alteration in mesoscale aggregate morphology, 
the static dielectric permittivity of the ionic liquid mixtures are increased by almost $100 \%$ relative to the neat ionic liquids.

The ability to tune $\varepsilon_{s}$ is significant since the vast majority of aprotic ionic liquids have low to moderate values of around 7-15, typical of low polarity solvents. [57-60] Higher $\varepsilon_{s}$ values are expected to influence ionic liquid/solute and ionic liquid/solid-surface interactions with potentially critical implications for the application of ionic liquids in biomass processing, chemical synthesis, nanoparticle growth and as electrolytes in solar cells, batteries, and super-capacitors. [1, 7, 61, 62] The substantial increase in $\varepsilon_{s}$ of the ionic liquid mixtures and its direct link to aggregate morphology and dynamics provides a new route to tuning this important physical parameter. More generally, this study highlights the advantage of combining techniques capable of probing composition-dependent changes in mesoscale aggregate morphology as well as mesoscale aggregate dynamics.

J.S. acknowledges support by the National Science Foundation, the Division of Chemistry through No. CHE-1753282. T.C. gratefully acknowledges financial support from the U.S. Army Research Office under Contract No. W911NF-17-1-0052. The BDS, DMS, and DSC measurements were conducted at the Oak Ridge National Laboratory's Center for Nanophase Materials Sciences, which is sponsored by the Division of Scientific User Facilities, Office of Basic Energy Sciences, U.S. Department of Energy. U. K. and J. K. S. acknowledge the support by the National Science Foundation. the Divison of Chemical, Biomolecular, Environmental, and Transport through the grant CBET-1706978. The x-ray scattering experiments were performed at the Duke University Shared Materials Instrumentation Facility (SMIF), a member of the North Carolina Research Triangle Nanotechnology Network (RTNN), which is supported by the National Science Foundation (Grant No. ECCS-1542015) as part of the National Nanotechnology Coordinated Infrastructure (NNCI). Sincere thanks to Prof. Austen Angell for helpful discussions. Computational resources were provided by the High Performance Computing Cluster at Oklahoma State University.

* jsangoro@utk.edu

$\dagger$ jindal.shah@okstate.edu

[1] J. P. Hallettand T. Welton, Room-Temperature Ionic Liquids: Solvents for Synthesis and 
Catalysis. 2, Chemical Reviews 111, 3508 (2011).

[2] Z. Heand P. Alexandridis, Nanoparticles in Ionic Liquids: Interactions and Organization, Physical Chemistry Chemical Physics 17, 18238 (2015).

[3] M. Antonietti, D. Kuang, B. Smarsly, and Y. Zhou, Ionic Liquids for the Convenient Synthesis of Functional Nanoparticles and Other Inorganic Nanostructures, Angewandte Chemie International Edition 43, 4988 (2004).

[4] D. R. MacFarlane, N. Tachikawa, M. Forsyth, J. M. Pringle, P. C. Howlett, G. D. Elliott, J. H. Davis, M. Watanabe, P. Simon, and C. A. Angell, Energy Applications of Ionic Liquids, Energy \& Environmental Science 7, 232 (2014).

[5] M. H. Chakrabarti, F. S. Mjalli, I. M. AlNashef, M. A. Hashim, M. A. Hussain, L. Bahadori, and C. T. J. Low, Prospects of Applying Ionic Liquids and Deep Eutectic Solvents for Renewable Energy Storage by Means of Redox Flow Batteries, Renewable and Sustainable Energy Reviews 30, 254 (2014).

[6] M. Smiglak, J. M. Pringle, X. Lu, L. Han, S. Zhang, H. Gao, D. R. MacFarlane, and R. D. Rogers, Ionic Liquids for Energy, Materials, and Medicine, Chemical Communications 50, 9228 (2014).

[7] M. Armand, F. Endres, D. R. MacFarlane, H. Ohno, and B. Scrosati, Ionic-liquid Materials for the Electrochemical Challenges of the Future, Nature Materials 8, 621 (2009).

[8] D. J. G. P. van Osch, L. J. B. M. Kollau, A. van den Bruinhorst, S. Asikainen, M. A. A. Rocha, and M. C. Kroon, Ionic Liquids and Deep Eutectic Solvents for Lignocellulosic Biomass Fractionation, Physical Chemistry Chemical Physics 19, 2636 (2017).

[9] C. S. Santos, H. V. R. Annapureddy, N. S. Murthy, H. K. Kashyap, E. W. Castner, and C. J. Margulis, Temperature-dependent Structure of Methyltributylammonium bis(trifluoromethylsulfonyl)amide: X ray Scattering and Simulations, The Journal of Chemical Physics 134, 64501 (2011).

[10] C. S. Santos, N. S. Murthy, G. A. Baker, and E. W. C. Jr., Communication: X-ray scattering from Ionic Liquids with Pyrrolidinium Cations, The Journal of Chemical Physics 134, 121101 (2011).

[11] R. Hayes, G. G. Warr, and R. Atkin, Structure and Nanostructure in Ionic Liquids, Chemical Reviews 115, 6357 (2015).

[12] O. Russina, F. Lo Celso, N. Plechkova, C. J. Jafta, G. B. Appetecchi, and A. Triolo, Topics 
in Current Chemistry, Vol. 375 (2017) p. 58.

[13] J. N. A. Canongia Lopesand A. 1. A. H. Pádua, Nanostructural Organization in Ionic Liquids, The Journal of Physical Chemistry B 110, 3330 (2006).

[14] A. Triolo, O. Russina, H. J. Bleif, and E. Di Cola, Nanoscale Segregation in Room Temperature Ionic Liquids, The Journal of Physical Chemistry B 111, 4641 (2007).

[15] U. Kapoorand J. K. Shah, Globular, Sponge-like to Layer-like Morphological Transition in 1-n-Alkyl-3-methylimidazolium Octylsulfate Ionic Liquid Homologous Series, The Journal of Physical Chemistry B 122, 213 (2017).

[16] U. Kapoorand J. K. Shah, Effect of Molecular Solvents of Varying Polarity on the SelfAssembly of 1-n-Dodecyl-3-methylimidazolium Octylsulfate Ionic Liquid, Journal of Theoretical and Computational Chemistry 17, 1840004 (2018).

[17] J. C. Araque, J. J. Hettige, and C. J. Margulis, Modern Room Temperature Ionic Liquids, a Simple Guide to Understanding Their Structure and How It May Relate to Dynamics, The Journal of Physical Chemistry B 119, 12727 (2015).

[18] Q. Berrod, F. Ferdeghini, J.-M. Zanotti, P. Judeinstein, D. Lairez, V. García Sakai, O. Czakkel, P. Fouquet, and D. Constantin, Ionic Liquids: Evidence of the Viscosity Scale-Dependence, Scientific Reports 7, 2241 (2017).

[19] F. Ferdeghini, Q. Berrod, J.-M. Zanotti, P. Judeinstein, V. G. Sakai, O. Czakkel, P. Fouquet, and D. Constantin, Nanostructuration of Ionic Liquids: Impact on the Cation Mobility. A Multi-scale Study, Nanoscale 9, 1901 (2017).

[20] T. Burankova, G. Simeoni, R. Hempelmann, J. F. Mora Cardozo, and J. P. Embs, Dynamic Heterogeneity and Flexibility of the Alkyl Chain in Pyridinium-Based Ionic Liquids, The Journal of Physical Chemistry B 121, 240 (2016).

[21] B. Aoun, M. A. González, J. Ollivier, M. Russina, Z. Izaola, D. L. Price, and M.-L. Saboungi, Translational and Reorientational Dynamics of an Imidazolium-Based Ionic Liquid, The Journal of Physical Chemistry Letters 1, 2503 (2010).

[22] P. J. Griffin, Y. Wang, A. P. Holt, and A. P. Sokolov, Communication: Influence of Nanophase Segregation on Ion Transport in Room Temperature Ionic Liquids, The Journal of Chemical Physics 144, 151104 (2016).

[23] Y. Wangand G. A. Voth, Tail Aggregation and Domain Diffusion in Ionic Liquids, The Journal of Physical Chemistry B 110, 18601 (2006). 
[24] T. Sonnleitner, D. A. Turton, S. Waselikowski, J. Hunger, A. Stoppa, M. Walther, K. Wynne, and R. Buchner, Dynamics of RTILs: A Comparative Dielectric and OKE Study, Journal of Molecular Liquids 192, 19 (2014).

[25] D. A. Turton, J. Hunger, A. Stoppa, G. Hefter, A. Thoman, M. Walther, R. Buchner, and K. Wynne, Dynamics of Imidazolium Ionic Liquids from a Combined Dielectric Relaxation and Optical Kerr Effect Study: Evidence for Mesoscopic Aggregation, Journal of the American Chemical Society 131, 11140 (2009).

[26] M. D. Fayer, Dynamics and Structure of Room Temperature Ionic Liquids, Chemical Physics Letters 616, 259 (2014).

[27] T. Cosby, Z. Vicars, Y. Wang, and J. Sangoro, Dynamic-Mechanical and Dielectric Evidence of Long-Lived Mesoscale Organization in Ionic Liquids, The Journal of Physical Chemistry Letters 8, 3544 (2017).

[28] T. Yamaguchi, Coupling between the Mesoscopic Dynamics and Shear Stress of a Roomtemperature Ionic Liquid, Physical Chemistry Chemical Physics 20, 17809 (2018).

[29] O. Russina, F. Lo Celso, N. V. Plechkova, and A. Triolo, Emerging Evidences of MesoscopicScale Complexity in Neat Ionic Liquids and Their Mixtures, The Journal of Physical Chemistry Letters 8, 1197 (2017).

[30] O. Russina, M. Beiner, C. Pappas, M. Russina, V. Arrighi, T. Unruh, C. L. Mullan, C. Hardacre, and A. Triolo, Temperature Dependence of the Primary Relaxation in 1-Hexyl3-methylimidazolium bis $\{$ (trifluoromethyl)sulfonyl $\}$ imide, The Journal of Physical Chemistry B 113, 8469 (2009).

[31] M. Kofu, M. Nagao, T. Ueki, Y. Kitazawa, Y. Nakamura, S. Sawamura, M. Watanabe, and O. Yamamuro, Heterogeneous Slow Dynamics of Imidazolium-Based Ionic Liquids Studied by Neutron Spin Echo, The Journal of Physical Chemistry B 117, 2773 (2013).

[32] E. W. Castnerand J. F. Wishart, Spotlight on Ionic Liquids, The Journal of Chemical Physics 132, $120901(2010)$

[33] K. Shimizu, C. E. S. Bernardes, and J. N. Canongia Lopes, Structure and Aggregation in the 1-Alkyl-3-Methylimidazolium Bis(trifluoromethylsulfonyl)imide Ionic Liquid Homologous Series, The Journal of Physical Chemistry B 118, 567 (2014).

[34] K. Shimizu, M. F. Costa Gomes, A. A. H. Pádua, L. P. N. Rebelo, and J. N. Canongia Lopes, Three Commentaries on the Nano-Segregated Structure of Ionic Liquids, Journal of Molecular 
Structure: THEOCHEM 946, 70 (2010).

[35] M. Brehmand B. Kirchner, TRAVIS - A Free Analyzer and Visualizer for Monte Carlo and Molecular Dynamics Trajectories, Journal of Chemical Information and Modeling 51, 2007 (2011).

[36] C. E. S. Bernardes, K. Shimizu, A. I. M. C. Lobo Ferreira, L. M. N. B. F. Santos, and J. N. Canongia Lopes, Structure and Aggregation in the 1,3-Dialkyl-imidazolium Bis(trifluoromethylsulfonyl)imide Ionic Liquid Family: 2. From Single to Double Long Alkyl Side Chains, The Journal of Physical Chemistry B 118, 6885 (2014).

[37] K. Shimizuand J. N. Canongia Lopes, Probing the Structural Features of the 1-alkyl-3methylimidazolium Hexafluorophosphate Ionic Liquid Series Using Molecular Dynamics Simulations, Journal of Molecular Liquids 210, 257 (2015).

[38] K. Shimizu, A. A. Freitas, and J. N. Canongia Lopes, Structural Characterization of the [CnC1im][C4F9SO3] Ionic Liquid Series: Alkyl Versus Perfluoroalkyl Side Chains, Journal of Molecular Liquids 226, 28 (2017).

[39] M. Brehm, H. Weber, M. Thomas, O. Hollóczki, and B. Kirchner, Domain Analysis in Nanostructured Liquids: A Post-Molecular Dynamics Study at the Example of Ionic Liquids, ChemPhysChem 16, 3271 (2015).

[40] J. R. Sangoroand F. Kremer, Charge Transport and Glassy Dynamics in Ionic Liquids, Accounts of Chemical Research 45, 525 (2012).

[41] F. Pabst, J. Gabriel, and T. Blochowicz, Mesoscale Aggregates and Dynamic Asymmetry in Ionic Liquids Evidence from Depolarized Dynamic Light Scattering, The Journal of Physical Chemistry Letters 10, acs.jpclett.9b00686 (2019).

[42] T. Cosby, Z. Vicars, M. Heres, K. Tsunashima, and J. Sangoro, Dynamic and Structural Evidence of Mesoscopic Aggregation in Phosphonium Ionic Liquids, The Journal of Chemical Physics 148, 193815 (2018).

[43] P. J. Griffin, A. P. Holt, K. Tsunashima, J. R. Sangoro, F. Kremer, and A. P. Sokolov, Ion Transport and Structural Dynamics in Homologous Ammonium and Phosphonium-based Room Temperature Ionic Liquids, The Journal of Chemical Physics 142, 84501 (2015).

[44] C. Gainaru, M. Wikarek, S. Pawlus, M. Paluch, R. Figuli, M. Wilhelm, T. Hecksher, B. Jakobsen, J. C. Dyre, and R. Böhmer, Oscillatory Shear and High-pressure Dielectric Study of 5-methyl-3-heptanol, Colloid and Polymer Science 292, 1913 (2014). 
[45] T. Hecksherand B. Jakobsen, Communication: Supramolecular Structures in Monohydroxy Alcohols: Insights from Shear-mechanical Studies of a Systematic Series of Octanol Structural Isomers, The Journal of Chemical Physics 141, 101104 (2014).

[46] T. Hecksher, Communication: Linking the Dielectric Debye Process in Mono-alcohols to Density Fluctuations, The Journal of Chemical Physics 144, 4 (2016).

[47] S. Arrese-Igor, A. Alegría, and J. Colmenero, Multimodal Character of Shear Viscosity Response in Hydrogen Bonded Liquids, Physical Chemistry Chemical Physics 10.1039/C8CP04183K (2018).

[48] R. Böhmer, C. Gainaru, and R. Richert, Structure and Dynamics of Monohydroxy AlcoholsMilestones Towards Their Microscopic Understanding, 100 Years after Debye, Physics ReportsReview Section of Physics Letters 545, 125 (2014).

[49] T. Yamaguchi, M. Saito, K. Yoshida, T. Yamaguchi, Y. Yoda, and M. Seto, Structural Relaxation and Viscoelasticity of a Higher Alcohol with Mesoscopic Structure, The Journal of Physical Chemistry Letters 9, 298 (2018).

[50] S. Torquato, Random Heterogeneous Materials: Microstructure and Macroscopic Properties (Springer-Verlag, New York, 2002) p. 701.

[51] T. C. Choy, Effective Medium Theory (Oxford University Press, New York, 1999).

[52] H. Looyenga, Dielectric Constants of Heterogeneous Mixtures, Physica 31, 401 (1965).

[53] L. K. H. van Beek, Dielectric Behavior of Heterogeneous Systems, in Progress in Dielectrics, Vol. 7, edited by J. B. Birks (Heywood Books, London, 1967).

[54] M. H. Boyle, The Electrical-Properties of Heterogeneous Mixtures Containing an Oriented Spheroidal Dispersed Phase, Colloid and Polymer Science 263, 51 (1985).

[55] F. Kremerand A. Schönhals, Broadband Dielectric Spectroscopy (Springer, Berlin, 2003).

[56] M. Mizoshiri, T. Nagao, Y. Mizoguchi, and M. Yao, Dielectric Permittivity of Room Temperature Ionic Liquids: A Relation to the Polar and Nonpolar Domain Structures, The Journal of Chemical Physics 132, 164510 (2010).

[57] H. Weingärtner, The Static Dielectric Permittivity of Ionic Liquids, Journal of Molecular Liquids 192, 185 (2014).

[58] M.-M. Huang, Y. Jiang, P. Sasisanker, G. W. Driver, and H. Weingärtner, Static Relative Dielectric Permittivities of Ionic Liquids at 25 degrees C, Journal of Chemical and Engineering Data 56, 1494 (2011). 
[59] H. Weingärtner, Understanding Ionic Liquids at the Molecular Level: Facts, Problems, and Controversies, Angewandte Chemie-International Edition 47, 654 (2008).

[60] C. Wakai, A. Oleinikova, M. Ott, and H. Weingärtner, How Polar are Ionic Liquids? Determination of the Static Dielectric Constant of an Imidazolium-based Ionic Liquid by Microwave Dielectric Spectroscopy, The Journal of Physical Chemistry B 109, 17028 (2005).

[61] T. Kim, H. C. Kang, T. T. Tung, J. D. Lee, H. Kim, W. S. Yang, H. G. Yoon, and K. S. Suh, Ionic Liquid-assisted Microwave Reduction of Graphite Oxide for Supercapacitors, RSC Advances 2, 8808 (2012).

[62] M. V. Fedorovand A. A. Kornyshev, Ionic Liquids at Electrified Interfaces, Chemical Reviews 114, 2978 (2014). 